\title{
Schwannoma in an accessory branch of the posterior cord of the brachial plexus: a rare case report
}

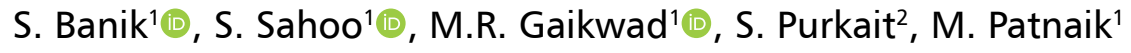 \\ ${ }^{1}$ Department of Anatomy, All India Institute of Medical Sciences, Bhubaneswar, Odisha, India \\ 2Department of Pathology, All India Institute of Medical Sciences, Bhubaneswar, Odisha, India \\ [Received: 28 April 2021; Accepted: 14 May 2021; Early publication date: 25 May 2021]
}

\begin{abstract}
Background: Variation in the posterior cord of the brachial plexus is complicated and creates a risky relationship with the neighbouring structures. This is of importance to the surgeons and anaesthetists who must deal with the region in surgeries and procedures. Moreover, any benign tumour like schwannoma is rare in the plexus comprising $5 \%$ of total head and neck schwannomas.

Materials and methods: We present a case of schwannoma of the brachial plexus in a cadaver during routine anatomy dissection for the medical students. The origin and order of branching of the posterior cord were recorded and photographs were taken. The tumour was present in an accessory branch of the posterior cord and removal was made in-toto. An immunohistochemistry study was done for confirmation of diagnosis.

Results: The classical branching of the posterior cord was present. Additionally, a branch existed that was supplying the triceps muscle and emerged directly from the posterior cord. Tumour having the dimension of $2 \times 1.8 \times 0.5 \mathrm{~cm}$ was present. Conclusions: Schwannomas are indolent but may cause compression of the nerve and resulting neurological symptoms. They might mimic nodules of supraclavicular fossa in breast carcinoma. Variations of the brachial plexus can also make the surgeons confused during surgery due to which anatomical knowledge of the possible variations is important. Pre- and post-operative complications can be easily predicted from it. Follow-up of the tumour is essential to track its progress and differentiation. (Folia Morphol 2022; 81, 3: 777-780)
\end{abstract}

Key words: brachial plexus, variation, schwannoma, breast carcinoma, posterior cord, triceps, nerve block

\section{INTRODUCTION}

One of the challenging structures a surgeon faces in neck and axillary region procedures is the brachial plexus. Formed by the anterior rami of the lower four cervical nerves and first thoracic nerve $(\mathrm{C} 5, \mathrm{C} 6$, $\mathrm{C} 7, \mathrm{C} 8$, and $\mathrm{T} 1$ ), the plexus often shows variations in distribution and arrangement of the root, trunk, division, or cord and even the branches, making this anatomical region extremely complicated. Variations are common at the junctions or sites of separation of individual parts, and collateral branches may arise from the trajectory of the entire plexus supplying scapular belt muscles [1].

Address for correspondence: Dr. M. Patnaik, Ass. Prof., Department of Anatomy, All India Institute of Medical Sciences, Bhubaneswar, Odisha, Sijua, Patrapada, 751019, India, tel: +91-8598030339, e-mail: madhumitapatnaik916@gmail.com

This article is available in open access under Creative Common Attribution-Non-Commercial-No Derivatives 4.0 International (CC BY-NC-ND 4.0) license, allowing to download articles and share them with others as long as they credit the authors and the publisher, but without permission to change them in any way or use them commercially. 


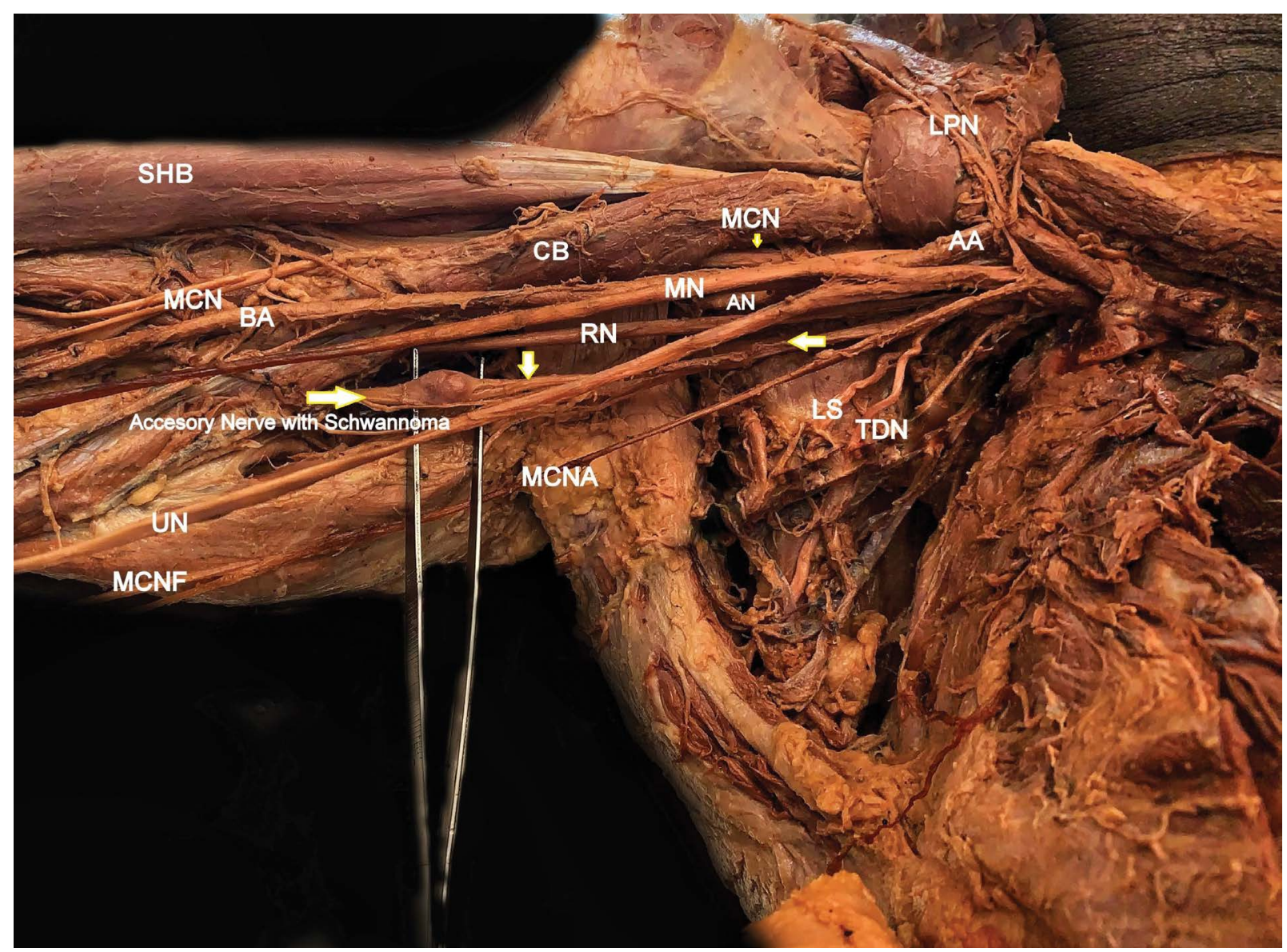

Figure 1. Dissected arm showing the brachial plexus with cords and branches. Notice the arrow that denotes the accessory branch arising out of the posterior cord of the plexus bearing the schwannoma; SHB — short head of biceps brachii; LPN — lateral pectoral nerve; AA — axillary artery; AN — axillary nerve; TDN — thoracodorsal nerve; $M N$ — median nerve; $L S$ - lower subscapular nerve; $M C N$ — musculocutaneous nerve; $\mathrm{CB}$ - coracobrachialis; RN — radial nerve; MCNA — medial cutaneous nerve of arm; UN — ulnar nerve; BA — brachial artery; MCNF — medial cutaneous nerve of forearm.

Apart from variations in anatomy, the brachial plexus is a rare site for harbouring $5 \%$ of the total $25-45 \%$ of the head and neck schwannoma, which is a benign, encapsulated, well-demarcated tumour arising from the Schwann cells of cranial, peripheral, or autonomic nerve sheaths [4]. It is mostly sporadic in origin and shows slow, insidious growth, and is often solitary [3]. In the living, it may present as a painless, palpable neck mass [7], or it may sometimes present as a supraclavicular cystic mass [3]. Due to proximity to vital structures, rarity and anatomic complexity of the brachial plexus, a schwannoma in association with a branching variation, is of utmost caution to the surgeons, anaesthetists for surgical procedures and anaesthetic blocks as well as to physicians to locate compression symptoms like pain, sensory loss, wakefulness, and paresis [1].

We present a serendipitous case of schwannoma arising from an accessory branch of the posterior cord of the brachial plexus in a cadaver.

\section{CASE REPORT}

During the routine anatomic dissection of the brachial plexus, a variation was found in the posterior cord on the right side. The classical branching of the posterior cord into the upper subscapular, thoracodorsal, lower subscapular, axillary nerve and its distal continuation as radial nerve was observed. What additionally present was a nerve emerging from the posterior cord, medial to the radial nerve and branching distally with two branches supplying the triceps muscle. At the distal end of this additional nerve, almost $16.50 \mathrm{~cm}$ from the outer border of the clavicle and $18.5 \mathrm{~cm}$ from the olecranon process, a well-encapsulated $2 \times 1.8 \times 0.5 \mathrm{~cm}$ sized round tumour was noticed (Fig. 1). The tumour was resected in toto, and sections were subjected to histopathological examination. Tumour was well-circumscribed and encapsulated (Fig. 2A). The tumour cells were arranged in short fascicles. The cells were having an 


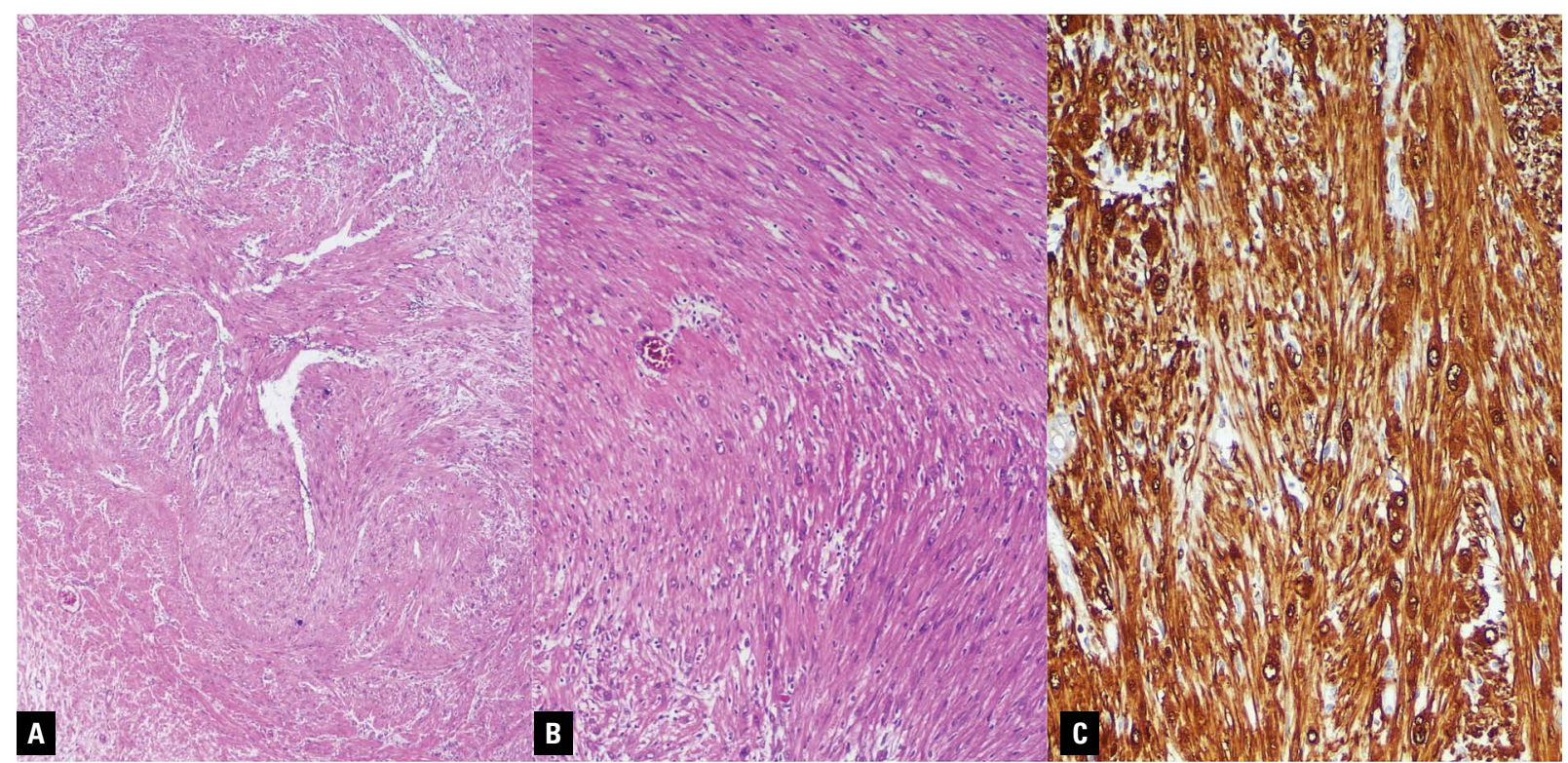

Figure 2. A. Representative photomicrograph showing a tumour which is well circumscribed and encapsulated; haematoxylin and eosin $(\mathrm{H} \& \mathrm{E}) \times 40$; B. The tumour cells are arranged in short fascicle. The cells have elongated vesicular nucleus and moderate fibrillary cytoplasm. No mitotic activity is seen; H\&E $\times 40$; C. The tumour cells showed strong immunopositivity for S-100; H\&E $\times 200$.

elongated vesicular nucleus and moderate fibrillary cytoplasm. No mitotic activity was seen (Fig. 2B). Immunohistochemical analysis of the tumour showed a strong, diffuse expression of S-100 positivity which confirmed it to be a schwannoma (Fig. 2C).

\section{DISCUSSION AND CONCLUSIONS}

Variation in the branching pattern of the posterior cord is common. Some existing studies have reported the classical branching pattern of brachial plexus in only $10.7 \%$ of cases [5]. The surgeons need to be aware of variant configurations of the cords of the brachial plexus while performing radical neck dissection and surgical exploration of the axilla and arm region to avoid damage to them. Moreover, the presence of communicating branches has to be kept in mind in the evaluation of unexplained sensory loss after trauma or surgical intervention in a particular area [8]. Neurotisation also needs proper identification of all the branches emerging from the posterior cord of the plexus. Entrapment neuropathy can be avoided if we know the plexus and its variations well enough [5]. The radial nerve can show variations in its origin, branching and communications. Abnormal communications of the radial nerve with the ulnar nerve have been reported in the plexus where the communicating branch was a separate filament given off from the radial nerve before entering the spiral groove where it did not mingle with the ulnar nerve fibres but entered the sheath and finally ended supplying the triceps muscle [6]. In our study, the accessory branch that had schwannoma in it was supplying the triceps muscle. The calibre of the nerve was like independent branches of the cord, arising a possibility that it could be a very high branching of the radial nerve itself. As observed by Uysal et al. [see 1], $53.5 \%$ of the foetus has variations in brachial plexus. The variations were mostly on the right-hand side, as stated by Luis Ernesto Ballesteros and Ramirez, which is consistent with our report [1]. Multiple schwannomas can happen, but most of them arise sporadically as single benign tumours [9], and in our case too, the tumour was solitary with the opposite side showing no presence of a tumour. The gross appearance of the tumour is oval or plexiform, and its colour may range from pink, tan, yellow to grey [4], which matched our findings where the tumour was oval and fixed along the long axis of the nerve with a mild pinkish tinge. The clinical importance of the schwannoma lies in the fact that it can cause direct nerve invasion; it can infiltrate the surrounding tissues or exert a local mass effect [9]. The symptoms may range from mild paraesthesia and numbness to direct tenderness and radiating pain. Preoperative sensory and motor deficit can be present, with the former being more common, almost $54 \%$ vs. $41 \%$ [9]. They are mostly benign but may mimic malignancies such as breast carcinoma with supraclavicular mass and metastatic 
lymph node [2]. Imaging studies like computed tomography scan and gadolinium-enhanced magnetic resonance imaging are important diagnostic techniques for preoperative evaluation of schwannoma [4]. However, surgery for schwannoma is indicated only when it causes neurological dysfunction or pain, compression symptoms and suspicions of malignan$c y$, but the real challenge lies in counselling patients about post-operative neurological deficits that are common. Despite easy enucleation of the tumour from the nerve, post-operative loss of sensation over the thumb or neurological phenomena due to $\mathrm{C} 5-\mathrm{C} 6$ severing has been reported [7]. Muscle transposition or primary nerve repair using nerve graft can prevent or reduce post-operative neurological symptoms. Brachial plexus schwannoma is a rare tumour entity, and coupled with an accessory branch of the posterior cord, it has immense importance in different branches of medicine as well as surgery and anaesthesia.

\section{Conflict of interest: None declared}

\section{REFERENCES}

1. Ballesteros LE, Ramirez LM. Variations of the origin of collateral branches emerging from the posterior aspect of the brachial plexus. J Brachial Plex Peripher Nerve Inj. 2007; 2: 14, doi: 10.1186/1749-7221-2-14, indexed in Pubmed: 17587464
2. da Costa Vieira RA, de Araujo Silva I, de Souza Coelho RD, et al. Brachial plexus schwannoma mimicking advanced breast carcinoma. Breast Dis. 2020; 39(2): 109-113, doi: 10.3233/BD-190432, indexed in Pubmed: 32083563.

3. Kho JP, Prepageran N. Huge brachial plexus schwannoma, masking as a cystic neck mass. AME Case Rep. 2018; 2: 41, doi: 10.21037/acr.2018.08.01, indexed in Pubmed: 30363802.

4. Kumar A, Akhtar S. Schwannoma of brachial plexus. Indian J Surg. 2011; 73(1): 80-81, doi: 10.1007/s12262-0100141-1, indexed in Pubmed: 22211049.

5. Muthoka JM, Sinkeet SR, Shahbal SH, et al. Variations in branching of the posterior cord of brachial plexus in a Kenyan population. J Brachial Plex Peripher Nerve Inj. 2011; 6: 1, doi: 10.1186/1749-7221-6-1, indexed in Pubmed: 21649927.

6. Natsis K, Giannakopoulou A, Piagkou M, et al. Connections between radial and ulnar nerve at high humeral level in cadavers: incidence, topography, and literature review. Surg Radiol Anat. 2018; 40(3): 313-322, doi: 10.1007/s00276-017-1939-3, indexed in Pubmed: 29124342.

7. Ranjan S, Arora N, Sethi D, et al. Schwannoma of the brachial plexus: a rare case report. Iran J Otorhinolaryngol. 2020; 32(111): 243-247, doi: 10.22038/ ijorl.2020.40635.2330, indexed in Pubmed: 32850513.

8. Rastogi R, Budhiraja V, Bansal K. Posterior cord of brachial plexus and its branches: anatomical variations and clinical implication. ISRN Anat. 2013; 2013: 501813, doi: 10.5402/2013/501813, indexed in Pubmed: 25969826.

9. Vučemilo L, Lajtman Z, Mihalj J, et al. Brachial plexus schwannoma - case report and literature review. Acta Clin Croat. 2018; 57(2): 366-371, doi: 10.20471/ acc.2018.57.02.19, indexed in Pubmed: 30431732. 